Fecha de recepción: mayo 2021 Fecha de aceptación: junio 2021 Versión final: julio 2021

\section{El diseño. Una herramienta de mediación, interacción y diálogo humano}

Leobardo Armando Ceja Bravo ${ }^{(*)}$

Resumen: El presente texto, busca contribuir a la reflexión sobre la interacción dado entre las personas a través de los objetos diseñados. De esta relación subyace el aporte de mediación que tiene el diseño, a saber, dialéctico. En este sentido, reflexionar sobre esta implicación que el diseño tiene, resulta crucial y más en los tiempos que corren en la actualidad. De igual forma, resultará fundamental analizar la influencia e intervención que el diseño tiene en todos los ámbitos de la vida, influyendo en la vivencia, en la experiencia y en las diversas formas de vida de las personas, propiciando, condicionando y determinando formas de vida y las maneras en cómo se relacionan con el entorno, por lo que es importante que se entienda a las disciplinas proyectuales como una forma condicionante de comportamientos humanos y sociales. En este contexto, la existencia de una "alfabetidad del diseño" (Lutnæs, 2019) es crucial en cuanto proceso de diálogo subyacente, es un continuum social. Pensar en el diseño como una disciplina orientada en formar, informar, propiciar, articular, regular, reordenar, resignificar o reinterpretar contribuye decididamente en la acción social implicada, dotando de valor y potencia a la interacción entre las personas. Para que este proceso dialógico se pueda dar, es importante tener presentes el contexto en el que dichas interacciones se dan, para ello, pensar en una "semiósfera" (Lotman, 1996) de las interacciones, es una oportunidad para analizar los diversos diálogos resultantes entre las personas y lo diseñado, produciéndose así un doble proceso "hermenéutico analógico" (Beuchot, 2016) el primero de ellos dado cuando el diseñador, realiza todas las acciones requeridas e implicadas para llegar a una respuesta de diseño, el segundo momento se da, una vez que lo diseñado se encuentra inmerso dentro del contexto social generando una interacción con las personas.

Palabras clave: Disciplina proyectual - alfabetidad - interacción - proceso dialógico - interpretación

[Resúmenes en inglés y portugués en la página 98 ]

(1) Profesor Investigador de Tiempo Completo en la Facultad de Diseño de la Universidad De La Salle Bajío (México). Doctor en Desarrollo y Docencia del Diseño por parte de la Universidad Madero en Puebla. Miembro del Sistema Nacional de Investigadores (SIN) Nivel: 1. Es autor de 4 libros y diversos artículos Areas de interés: Paradigma de la Complejidad, la investigación en diseño, las interacciones sociales y la investigación cultural y artística. 


\section{La semiosfera. Una representación de identificar las formas de la cultura}

La actualidad está caracterizada por la complejidad creciente. Ello es posible vivenciarlo en todos los ámbitos en los que el ser humano se encuentra inserto. De la misma forma, toda acción y actividad humana, estará influenciada y determinada por distintos niveles de implicación del diseño.

Si la actualidad está caracterizada por la complejidad, ello se encuentra directamente relacionado con el diseño, a saber, es gracias a la "configuración del mundo de lo artificial" (Simon, 1996). Asimismo, es posible afirmar la existencia de una sobresaturación de estímulos, entendiéndose como exceso de información teniendo como efecto la reducción de significado.

Buscando entender la configuración de los espacios que se han configurado a través del tiempo, se precisa entender las múltiples relaciones que el ser humano ha establecido entre las mismas personas, pero también con diversas especies, ello a través de la integración de múltiples medios, recursos y soportes, los cuales influyen en mayor o menor medida en la vida cotidiana. Bajo esta perspectiva es posible hablar de la existencia de una "infoesfera" (Berardi, 2017, p. 204).

Lo que se intenta poner en contexto es la extrema complejidad de la vida actual, en medio de la cual vivimos, pues se puede relacionar con las diversas áreas del diseño, sus respectivos enfoques y finalidades en un entramado que cruza todos los ámbitos de la forma de vida en la que nos encontramos. Por tanto, pensar en el impacto e influencia que el diseño tiene y ejerce en el día a día, puede seguir considerándose como una necesidad reflexiva fundamental.

El énfasis inicial en el que se vincula el diseño, podrá encontrarse e identificarse en los diversas formas y estilos de vida, pero también en el sentido del gusto, en las expresiones artísticas, musicales, con los objetos como mobiliario, ropa, alimentación, educación, configuración espacial que se viven, disfrutan, socializan y por consiguiente, generan procesos de interacción, vivenciadas y trasladadas al ámbito personal de las experiencias.

En medio de esta trama esbozada, se precisa que aproximarse a los diversos niveles de significación implícitos, la semiótica de la cultura (Lotman, 1996) a través de la semiosfera se erige como la búsqueda de la interpretación de un espacio simbólico de interacción configurado.

El continuum comunicativo implícito y ejercido cotidianamente en un contexto determinado, por un grupo de personas que de diversas formas interactúa, genera, produce, recibe e intercambia información. Este intercambio de información está dándose de diversas formas, con igualmente diversas finalidades, tanto de modo individual como de manera colectiva, siendo una evidencia más del grado de complejidad imperante y creciente.

En el actual contexto de pandemia que se sigue viviendo, muchas desigualdades se han hecho evidentes, agudizando múltiples problemáticas y otros diálogos subyacentes los cuales surgen en tanto búsqueda de dotar de sentido a las condiciones de movilidad, comunicación e interacción implicadas haciendo explícita la proliferación de recursos digitales en el mejor de los casos, porque también se ha mostrado el contexto de precariedad creciente en la que una gran cantidad de personas viven. 
Las interacciones significativas dadas dentro del contexto de la cultura, es decir, dentro de una semiosfera determinada, implica la yuxtaposición de lenguajes de naturaleza distinta, conviviendo en un mismo tiempo. Este hecho, es una muestra más de la complejidad creciente e imperante en todo contexto, por tanto, la pluralidad de lenguajes, la generación de diversos procesos de significación, ejercidos por agentes sociales con diversos propósitos, requieren de un estudio y aproximación particular, puesto que los múltiples significados subyacente puede contribuir en facilitar u obstaculizar cualquier intensión comunicativa. El diseñador debe estar claro del tipo de mensajes implicados dentro del objeto de diseño generado, y si no es posible determinar en su totalidad las implicaciones comunicativas que éste tendrá dentro de un contexto social, determinado, entonces se podrá establecer que no ha analizado por completo los códigos y mensajes implicados, por lo que el diálogo propuesto, podrá tomar otros cauces y no los deseados.

Otra de las implicaciones comunicativas relacionadas con lo diseñado estará dada en lograr que se reconozcan las "funciones y usos sociales de los sistemas de significado" (Coronado y Hodge, 2017, p. 66).

Lo diseñado, dentro de un contexto social, interactúa en mayor o menor medida con grupos diversos, lo cual producirá procesos interpretativos que pueden ser distintos. Las posibles lecturas que del emanen podrán o no establecer múltiples intercambios que puedan ser significativos. Si estos intercambios se dan de la forma esperada, entonces se han puesto en marca diálogos significativos. En este sentido habrá que entender que tanto el objeto diseñado, como la comprensión del mismo, es posible gracias a la interacción social imperante, y ello supone interpretar al objeto diseñado como "un texto" (Bajtín (1989, Lotman, 1996) en cuya "lectura de la realidad" (Freire, 2011) le confiere a todas las personas la posibilidad de configurar una cierta noción de lo que lo diseñado representa. Se podría decir que todo objeto diseñado, una vez que se encuentra dentro del contexto social, y al momento de estar expuesto, propicia entrecruces transversales, es decir, puede ser identificado por distintos grupos sociales, quienes podrán interpretarlo en mayor o menor medida de forma similar, pero en la medida en la que logra empatar su sentido con lo que las personas interpretan, entonces se podrá decir, que lo diseñado, conllevará un efecto deseado, a saber, organizar, orientar, regular o activar ciertas acciones y conductas deseadas. Esta doble influencia establecida mediante la flexibilidad adjudicada tanto al código como a los mensajes, hace explícita la forma de entender el sentido de las prácticas cotidianas, lo que Wittgenstein (2008) denomina “...los juegos de lenguaje y formas de vida..

Uno de los aspectos fundamentales que tiene la identificación de una determinada semiosfera, es la identificación de la relación adentro-afuera. Esta dicotomía está caracterizada por la identificación de los textos y subtextos imperantes. Plantearlo en estos términos, automáticamente logra identificar la existencia de un afuera, es decir, una serie de texto inteligibles que por esa condición serán ajenos a un determinado contexto, pero pertenecientes a otro.

En estos casos, en la que los objetos diseñados parecen no estar en conexión con las personas, habrá que revisar el contenido significativo que se espera que tenga, ya que, estarán requiriendo otra clase de signos-textos que lo resignifiquen. 
Al momento de identificar los textos implicados dentro de una semiosfera, se hará patente la existencia de una diferencia, es decir, el proceso de interpretación llevado a cabo dentro de un contexto, estará resultando pertinente en la medida en la que la interpretación de los signos-textos resultantes sean reconocidos, en este sentido, quedarán excluidos aquellos que no sean significativos.

Esta pertenencia a otros sistemas de significación, podrán entenderse como procesos dinámicos de aculturización, por lo que una gran cantidad de influencias, cuyo origen está alejado a una realidad contextual, contribuye al desarrollo de estos procesos.

Estos procesos se producen todo el tiempo. En los tiempos que corren actualmente es más fácil identificarlos dentro de un contexto de uso tecnológico. Esta posibilidad comunicativa, dada por los dispositivos digitales genera un proceso de interacción e interrelación comunicativa, propiciando un flujo comunicativo.

Los traslados comunicativos se producen todo el tiempo. Provocando el intercambio significativo y por tanto un proceso dialógico continuado, por lo que pensar en que dichos tránsitos comunicativos se están llevando acabo, presuponen al mismo tiempo un "acoplamiento estructural" (Luhmann 2007), es decir, la sincronización comunicativa de algunos elementos significativos, ajenos al signo-texto, que son provenientes de otra semiosfera, y que en su proceso de interacción logran ser interpretados para enlazar o vincular a una o más personas.

\section{La influencia e injerencia del diseño en todos los ámbitos de la vida. Vivencias y experiencias}

El esquema 1. sintetiza aspectos generales abordados en el apartado anterior, asimismo, permite una vista general al complejo desarrollo propuesto y a las interrelaciones que subyacen. Se logra identificar la existencia de una interior y un exterior, caracterizados por la idea de semiósfera y contexto, asumiendo que al exterior de ella existirá otra que contenga elementos similares aunque con una significación distinta, razón por la cual es parte de otro contexto.

La actividad del diseño, históricamente se ha encargado de transformar los recursos que existen en la faz de la tierra para servir de materia e insumo, que mediante diversas acciones con las que se logra transformar en objeto. Esta búsqueda y entendimiento de lo que la materia es, así como de las posibilidades de transformación le ha implicado al ser humano, un arduo proceso de experimentación, de conocimiento y de intentos fallidos. El éxito de dicha empresa, ha constituido una de las labores, en cuyo empeño, el tiempo ha fraguado generación tras generación de hacedores del mundo material que nos rodea, a saber, de objetos configurados por los diseñadores. Son ellos quienes se han empeñado en trasformar la materia en comodidad, emoción, necesidad y adecuación. Haciendo tangible aspectos como los ligados a las emociones, ya que son estas las que "...reflejan nuestras experiencias, asociaciones y recuerdos personales.” (Norman, 2005, p. 63). 


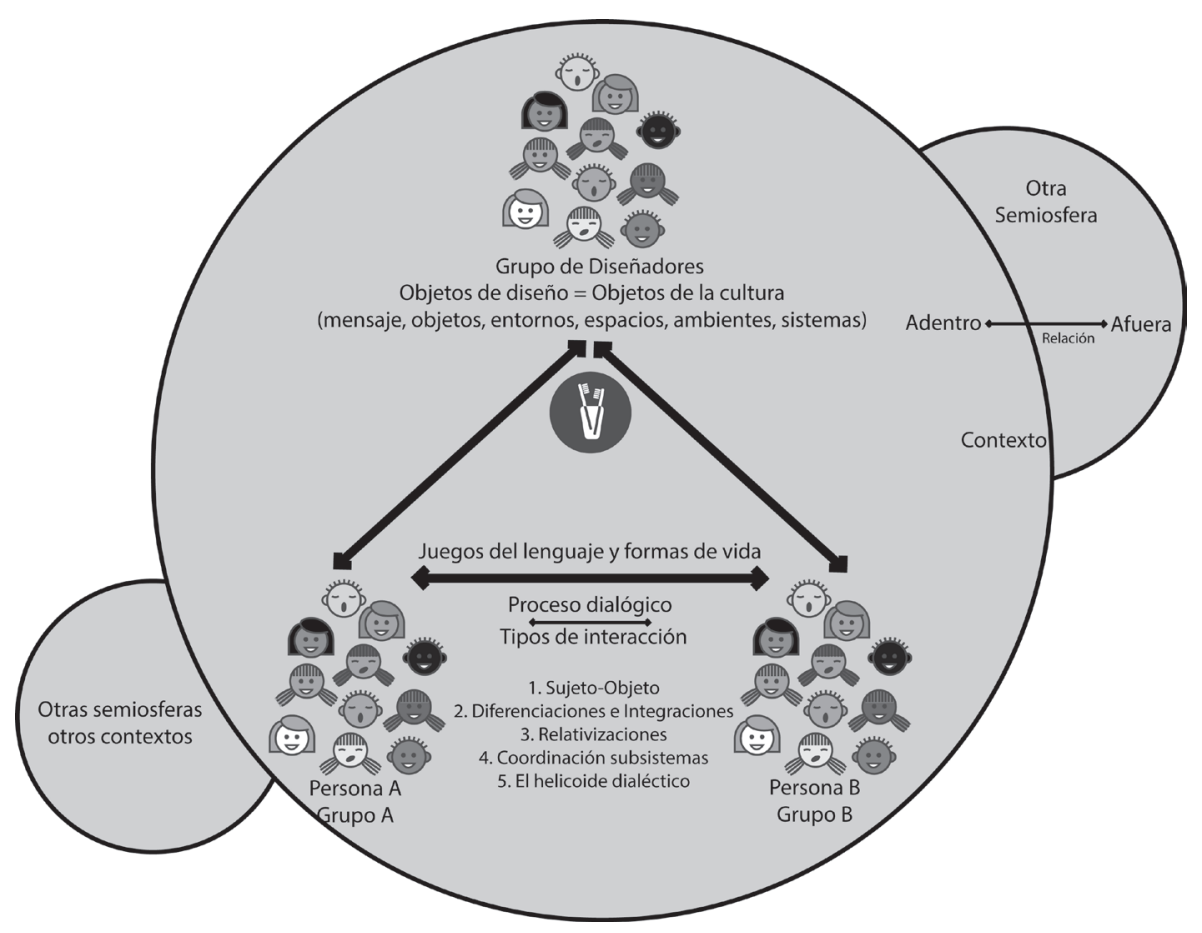

Esquema 1. Abstracción conceptual de elementos para complejizar las relaciones dialógicas.

Elaboración personal, 2021.

El propósito transformador de la materia, en la cual se ha empeñado el diseñador en ejercer, ha exigido que la experimentación se convierta en experiencia y que la experiencia se torne en una idea de progreso. La práctica se hace presente en la medida en la que el empeño en entender y modificar las propiedades de la materia la consagren en un nuevo objeto. Dicho objeto, deberá entenderse desde distintos puntos de vista, a saber, material, funcional, estético y simbólico.

Como punto de partida, resultará por demás estimulante reflexionar sobre la fuerza de las experiencias en el proceso de configuración de los objetos. Ello a razón de que la experiencia, de acuerdo con Victor Margolin (2005) “...el verdadero aprendizaje proviene de una inmersión total en la experiencia.” (p. 98) evidencia un continuum implicado en el devenir de la configuración de objetos. Ya que como lo refiere Victor Margolin, “...la experiencia puede volverse tanto más rica y profunda cuanto mayor sea el grado de conciencia 
y comprensión que tengamos del pasado." (Margolin, 2005, p. 62). De lo anterior puede establecerse la existencia de una tradición vinculada con el hacer el objeto, y con ello, es posible aproximarse a los múltiples niveles de reflexión implicados.

El objeto de diseño conjunta en su posibilidad de ensamblaje (Latour, 2008), efectos tangibles e intangibles-de adaptación y regulación. Una vez que se logra entender el funcionamiento del objeto diseñado, la adaptación y uso cotidiano deviene en sentido.

El efecto regulador que implica la comprensión del objeto de diseño da la pauta para que el hábito se instaure y con él, la adaptación simbólica del objeto de diseño a la vida cotidiana. Este efecto de adaptación rutinario, provoca que la reflexión sobre el objeto de diseño pierda su condición de novedad, es decir, de la de un objeto problemático-funcional dando lugar a la incorporación cotidiana. Solo se volverá latente, cuando se evidencia algún aspecto funcional, dado mediante la ruptura del hábito.

Todos los ámbitos de la vida humana, han sido influenciados, modificados e integrados a una forma de comportamiento, comodidad y formas de socializar. Ello ha sido gracias a las disciplinas proyectuales, las cuales han sido producto de múltiples procesos de entendimiento y transformación del entorno. Así, las múltiples tareas con las que el diseño puede ser asociado, implica revisar todas las actividades que se realizan, tanto de forma individual como de forma grupal. Este reconocimiento, requiere entender al diseño como una actividad esencialmente ontológica. Para Arturo Escobar (2016, p. 12) "El diseño es ontológico porque cada objeto, herramienta, servicio o, incluso, narrativa en los que está involucrado, crea formas particulares de ser, saber y hacer."

\section{Un continuum social de interacción y aprendizaje no formal dado gra- cias a la Alfabetidad del diseño}

La noción de movimiento y transformación está presente en un continuum, bajo esta idea habría que entender al diseño como una disciplina en cuyas acciones se estarían centrando actividades orientadas a propiciar una acción, un cambio, una ruptura con lo habitual o la permanencia de hábitos, y también puede estar orientada hacia la regulación, la toma de conciencia o de acción, buscando trastocar lo establecido, intentando el cambio y la adecuación de las personas a circunstancias diversas, concretas y particulares; bajo este contexto el diseño se encuentra inserto en una lógica asociada al cambio, al movimiento y a la transformación social.

Ahora bien, la relación que se establece entre los estímulos exteriores, llamada realidad, y los procesos de interpretación internos, da como resultado, el proceso de semiosis, es decir, "es el proceso por medio del cual se establece una relación entre significado y significante" (Albano, Levit, y Rosenberg, 2005 p. 206), la significación y sentido que dicho estímulo exterior tiene sobre la persona que percibe es de gran importancia, puesto que es una forma potente por medio del cual las personas se pueden relacionar e interactuar con los objetos diseñados. En ese tenor, los cinco sentidos todo el tiempo están percibiendo estímulos del exterior, por lo que el proceso de interpretación es un continuum. 
Este continuum puede evidenciarse de muy diversas formas, para algunos autores como Vygotsky (2015), se da gracias a la mediación del lenguaje. Aproximarse a la percepción de las personas, implica re-semiotizar el sentido que dichos objetos de diseño tienen en tanto portadores de cultura y por ende, de significado.

Aproximarse al significado atribuido a ciertos objetos de diseño configurados en un contexto determinado, constituyen un ejercicio de semiosis de carácter complejo. Esta complejidad abarca el carácter multidimensional humano (Morin, 1998), esto es, buscar aproximarse al entendimiento de los procesos que vinculan al propio ser humano, en relación con el vínculo que establecen con el exterior en relación con el interior. Esta conexión dada entre interior personal-exterior contexto, también contribuye al desarrollo de interacciones cuya finalidad estará vinculada con la vivencia y experiencia cotidiana que toda persona tiene, generando diversas formas de aprendizaje.

Entender las vivencias y experiencias humanas, bajo una perspectiva de aprendizaje, implica analizar la forma de interacción con los objetos diseñados.

En el contexto tecnológico en el que actualmente se está viviendo, es innegable el efecto que ello ha tenido en la cotidianidad de las personas e implícitamente ha conllevado a la necesidad de afrontar procesos de adaptación, aprendizaje e interacción nuevos para muchos y con diversos niveles o alcances, esta circunstancia ha podido contribuir a reflexionar sobre la forma en la que las personas entienden, se relacionan y familiarizan con nuevos y diversos objetos tecnológicos diseñados, dejando al descubierto los procesos de aprendizaje, apropiación y ejecución vinculados con su uso y aplicación. Entender esta forma de aprendizaje como evidencia de un proceso de semiosis, hace evidente la existencia de posibles diálogos subyacentes y en cuya significación se pueden encontrar diversos valores y propiedades específicas.

En ese sentido, resulta pertinente tomar en consideración lo que Han (2018, p. 29) afirma, al referir que: "La globalización es un fenómeno complejo. No hace simplemente desaparecer la variedad de signos, ideas, imágenes, condimentos, olores. La producción de unidad y de monotonía no es característica ni de la naturaleza ni de la cultura.."

La alfabetidad está relacionada con los aportes humanos en los que todo "...individuo participe en la vida diaria y en la sociedad.” (Lutnæs, 2019, p. 1303). En este sentido, para pensar en este proceso de aprendizaje personal, es fundamental considerar las implicaciones del diseño como un recurso de mediación, es decir, como un recurso que le permite a la persona hacer evidentes diversas acciones, conductas, procedimientos o interacciones, que tendrían que implicar de una forma u otra la adquisición de nuevos saberes, habilidades y destrezas, teniendo necesariamente una implicación de reconfiguración de los esquemas mentales de reinterpretar la realidad circundante.

La ejecución de una doble acción interpretativa "hermenéutica análoga" (Beuchot, 2016), llevada a cabo en distintos momentos y que se encuentra vinculada al objeto de diseño, son dos aspectos fundamentales a tener presente, si lo que se busca es reflexionar sobre los procesos dialógicos implicados dentro del contexto social en el que interactúan, tanto los objetos como y la interacción de las personas.

Toda la serie de procesos que desarrolla el diseñador, en la búsqueda de configurar un objeto de diseño, implica que se realice un primer proceso de interpretación y el resultado 
de toda esta serie de procesos, podría ser considerado como un "análogo" es decir, un símil, ya que ante todo, el objeto diseñado es la representación de algo más. El segundo momento del proceso de interpretación está constituido por el esfuerzo interpretativo de la persona, toda vez que el objeto diseñado se encuentra inserto en la cultura.

Lograr entender, en este sentido una propiedad emergente que tiene lo diseñado, dentro del contexto social puede abrir una línea de estudio, reflexión, teorización y aplicación. Lo emergente, entonces puede ser abordado desde el enfoque centrado en los estudios en alfabetización (New Literacy Studies) en los que, en términos generales, se entiende “...la alfabetización como un medio de transformación social para los individuos y sus comunidades..." (Bravo y Bohemia, 2020, p. 2).

La incidencia del diseño en la transformación social requiere de la conciencia de todos sus actores, y radica en la búsqueda de la generación de un bien común mayor.

El devenir histórico del diseño ha demostrado que, son los propios diseñadores quienes han asumido roles, funciones y el sentido de su propia actividad, en función a las situaciones del contexto imperantes; antes que como parte de una convicción disciplinar genuina y ajena a las fuerzas que ejercen las circunstancias de su tiempo. Es decir, los diseñadores han respondido a las necesidades propias del contexto más bien de forma reactiva y no de forma propositiva.

\section{Interacción de las personas a través de los objetos diseñados. Un aporte dialéctico}

Gestionar el diálogo a través de los objetos de diseño, es buscar lograr procesos de interacción comunicativos en los que "la reciprocidad y la mutualidad" (Lotman, 1996, p. 18) se hagan presentes, suponiendo asimismo, una pertinencia temporal asignada al objeto de diseño.

Todo objeto diseñado será un análogo de la realidad a la que representa o alude, en ese sentido se podrá entender como un recurso de "mediación formal" (Ceja, 2018, p. 96), y es justamente en este entendido en medio del cual se puede identificar la vinculación dialógica que implícitamente marca a todo objeto diseñado. Asimismo, y dentro de un carácter práctico, cotidiano y vivencial, valores asociados con el conocimiento podrán relacionarse con el aporte alfabetizador del objeto de diseño, los cuales llevan implícitos actos de participación y diálogos con los que se pueden orientar esfuerzos, lazos y afectos comunicativos. En este mismo sentido, “...la alfabetización en diseño se encuentra emparentada con la formación de un tipo de ciudadanía, centrada en el diálogo y la participación democrática." (Vargas y Bride, 2020, p. 69).

Otro de los aportes significativos que se le puede adjudicar al efecto que mediación, interacción e interconexión dado, a través de lo diseñado, estriba en lo que Alva Noë (2010, p. 101) ha descrito como la conexión y la contigüidad, ya que "...ellas mismas trazan la coordinación y el destino común." 
La capacidad y fuerza humana se orientan como ejercicio reflexivo constante y de transformación de la materia en objetos, a través de la aplicación de diversos procesos igualmente diseñados. Esta reflexión y transformación de la materia en objetos diseñados, evidencian la cultura en medio de la cual subyace el sentido. Es, en esta búsqueda de sentido, que el proceso dialógico puede oscilar de un acto a otro buscando encarnar el sentido y articularse mediante el lenguaje.

La consideración anterior es importante porque logra hacer evidente que existe una consciencia y una intención de actuar ante las problemáticas imperantes en la actualidad. Asimismo, es posible afirmar que los participantes han demostrado una conciencia social, misma que se expresa mediante la dignificación de sus afirmaciones, es decir, que sus respuestas hacen evidentes "... una posición en la sociedad. Existir es reflejarse en la mirada del otro.” (Antaki, 2006, pp. 229-230).

La interacción que se establece entre las personas y lo diseñado contribuye a un diálogo subyacente, el cual resulta fundamental para la obtención de una interpretación acorde con el requerimiento de configuración implicado, y ello no es poca cosa, ya que este hecho ha contribuido en regular, propiciar, articular, reordenar, resignificar o reinterpretar la acción social implícita, en ese sentido “...el diálogo pasa a ser una exigencia epistemológica para una vivencia socialmente comprometida y cuya reflexión, compartida colectivamente, genera autorías múltiples." (Souza de Freitas, 2015, p. 35).

El esquema 2, es una abstracción conceptual de la argumentación llevada a cabo hasta el momento en el presente trabajo, pero al mismo tiempo, permite afinar y puntualizar algunos detalles que consideramos importante enfatizar.

En este sentido, se busca reflexionar sobre las diversas formas de interrelación dialógica que se pueden establecer (líneas punteadas). Los diversos procesos dialógicos subyacentes se pueden identificar de acuerdo con Rolando García (2000) en 5 posibles interacciones. La relación sujeto-objeto, puede constituirse como la primer forma de acción que se establece cotidianamente. (ver en el esquema 2, el número 1). El proceso dialógico implicado estará asociándose a los diversos “procesos de asimilación” (García, 2000, p. 131), es decir, implica la identificación de esquemas de acción, pero también de la conceptualización, que las personas establecen en la medida en que se relacionan con diversos objetos diseñados, modificando su conducta, comportamiento o acciones esperadas y asociadas. Esta relación sujeto-objeto, en su proceso de entendimiento y alfabetidad, logra introducir aspectos comportamentales y conductuales que las personas estarán incorporando a su actividad cotidiana de forma paulatina, modificando así, dinámicas tanto personales como de interacción social.

La transformación de ciertos aspectos análogos dentro del proceso de entendimiento, adaptación y ejecución dados entre las personas y los objetos diseñados, puede conducir al desarrollo del proceso dialógico identificado a partir de las "diferenciaciones e integraciones" (García, 2000, p. 131), caracterizado en el esquema 2 con el número 2.

Cuando todo el proceso de resolución de problemas se trabaja considerando únicamente el ámbito disciplinar y lo que ocurre dentro del ámbito de expertos, bien podrá catalogarse bajo la forma dialógica de lo que Rolando García (2000) ha denominado "relativizaciones", 
ya que se caracteriza “...cuando son considerados en forma aislada, se presentan como propiedades absolutas, entran luego en un juego de interdependencias dentro de los cuales las propiedades se tornan relativas..." (p. 131) misma que se encuentra representada con el número 3.

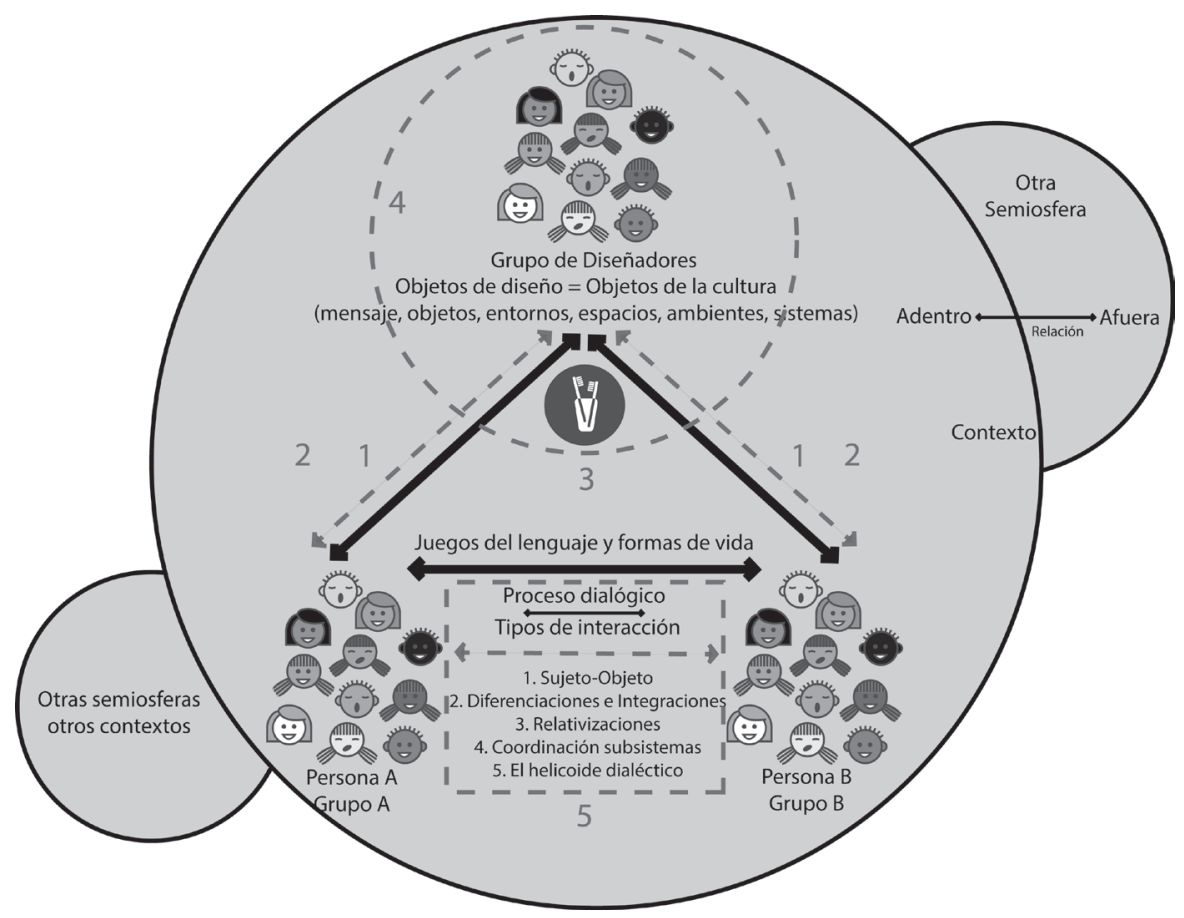

Esquema 2. Abstracción conceptual de elementos para complejizar las relaciones dialógicas y los tipos de diálogos subyacentes. Elaboración personal, 2021.

En lo concerniente al propio ámbito de los diseñadores se podría ejemplificar con el número 4, a los modos de acciones dialécticas implicados. Al considerar que al interior de la disciplina del diseño existen diversas áreas, enfoques, y experiencias particulares y que de cierta forma podrán ser interdependientes, se requiere de una coordinación para lograr articular estos elementos. Este hecho en sí mismo, constituye un proceso "dialéctico de mutua redefinición que los lleva a constituir una nueva totalidad dentro de la cual funcionan como subsistemas" (García, 2000, p. 131), y que el propio autor lo refiere como "Coordinación de subsistemas". 
La última forma dialéctica implicada, estará formulada bajo el nombre de "El helicoide dialéctico" (García, 2000), la cual estaría caracterizada por diversas interdependencias y "...la dinámica de las interacciones comprende necesariamente un aspecto de sucesión tal que todo proceso en el sentido de la construcción proactiva provoca reorganizaciones..." (García, 2000, pp. 131-132). Bajo esta última descripción es que se puede comprender la importancia que tienen los objetos de diseño, una vez que se encuentran insertos dentro del contexto social, haciendo evidente su capacidad para la mediación, la interrelación, la significación y la alfabetidad a la cual responderán, interactuarán y resignificarán las más diversas circunstancias cotidianas de las personas, mismo que queda caracterizado con el número 5 en el esquema 2 .

El proceso dialógico que se establece entre las personas y entre las personas y los objetos de diseño debe considerar que: “...por una parte admitimos que lo sensible no existe sino como relación de un sujeto con el mundo; pero por otra parte consideramos que las propiedades matematizables del objeto están exentas de la constricción de dicha relación, y que están efectivamente en el objeto tal como las concibo, tenga yo relación con dicho objeto o no." (Meillassoux, 2015, p. 26).

Quentin Meillassoux (2015) habla de propiedades que denomina matematizables. Retoma el planteamiento de demostración geométrica de Descartes, en el que establece que el carácter material de las cosas estarán dadas por las propiedades materiales y éstas pueden demostrarse por sus características del largo, ancho, profundidad, movimiento, figura y tamaño. En ese sentido, Wucius Wong (1991) lo refiere en los términos de los fundamentos del lenguaje visual. En este sentido, el diálogo se establece gracias a mediación del objeto de diseño, pudiendo hacer evidente e identificable "un lenguaje formal específico" (Meillassoux, 2015, p. 28).

\section{Reflexiones finales}

Bajo las condiciones en las que nos encontramos en la actualidad, reflexionar sobre las implicaciones del diseño y de sus acciones resultantes, en definitiva conlleva al reconocimiento de una ontología del diseño en la que el: “...'ser’ el mundo significa transformar y re-transformar el mundo, y no adaptarse a él. Es indudable que, como seres humanos, nuestra principal responsabilidad es intervenir en la realidad y mantener viva la esperanza." (Freire, 2015, p. 44) y ello es un lujo que puede permitir el diseño. Ahora bien, cuando se habla de lujo se hace en términos de posibilidad, es decir, la posibilidad de reflexionar, intervenir, modificar o transformar la realidad y buscar contribuir a la generación de mensajes, objetos, entornos, espacios, ambientes o sistemas, que incrementen la calidad de vida de las personas. Cuando ello se logra, se contribuye a la construcción de puentes de comunicación respetuosos que integran realidades que de origen pudieran estar fragmentadas o desconectadas y que gracias al diseño promulgan diálogos fructíferos. 
Uno de los efectos que hay que tener presente de la vida actual, está orientado a la creciente intención de crear experiencias. Más allá del uso común del término, es preciso reflexionar sobre la implicación que tiene esta intención. De acuerdo con Franco Berardi (2017, p. 204) al afirmar que: "La aceleración de la experiencia provoca una conciencia reducida de los estímulos, una pérdida de intensidad que concierne a la esfera de la estética, de la sensibilidad y también a la ética." El efecto entrópico que conlleva la proliferación de experiencias, como forma de vida, es el incremento del estrés, de la ansiedad y de un nivel de tensión incremental.

Entender la forma en la que las personas vivencian la realidad a través de lo diseñado es de gran importancia, puesto que ponen de manifiesto las diversas maneras de entender e interpretar el mundo y la relación que se puede establecer, mediante la contingencia de lo habitual; sin embargo se producen en un continuum yuxtapuesto que, - de la misma forma en la que lo refiere Flusser (2002) acerca del carácter conflictivo, y paradójicamente de ajuste y ordenamiento dado por los objetos diseñados-, se pueden establecer diversos diálogos, en los cuales se pueden evidenciar las diversos modos de percibir y vivenciar la realidad. "Diseñar para la conexión humana se refiere a diseñar para las relaciones que unas personas construyen con otras, considerando también su impacto en lo no-humano." (Barón y Echavarría, 2020, p. 110).

La relación que se establece entre objeto diseñado y persona, constituye un vínculo subjetivo cargado de sentido. Analizarlo, podrá evidenciar un tipo de diálogo que se entabla y que se muestra en diversos niveles como el perceptivo, emocional o racional y constituye un esfuerzo necesario para aproximarse a su entendimiento.

\section{Bibliografía}

Albano, S., Levit, A., y Rosenberg, L. (2005) Diccionario de semiótica, Buenos Aires, Argentina: Ed. Quadrata.

Antaki, I. (2006) El manual del ciudadano contemporáneo, México: Ed. Booket.

Bajtín, M. (1989) Teoría y estética de la novela, Madrid, España: Ed. Taurus.

Barón, M., y Echavarría, M. (2020) Laboratorio de empatía: indagando principios éticos para la educación en diseño, en Diseña, Agosto 2020, pp. 106-127.

Beuchot, M. (2016) Hechos e interpretaciones. Hacia una hermenéutica analógica. México: Ed. F.C.E.

Berardi, F. (2017) Fenomenología del fin. Sensibilidad y mutación conectiva, Buenos Aires, Argentina: Ed. Caja Negra.

Bravo, Ú., y Bohemia, E. (2020) Alfabetización en Diseño para Todos, en RChD: creación y pensamiento, 5(8). 63-76. DOI: 10.5354/0719-837X.2020.57649.

Ceja. L. (2018) La complejidad dentro del proceso de validación en el diseño. Una expresión subyacente del modelo funcional en Educación, Aplicación e Innovación en Diseño, México: Ed. Qartuppi. 
Coronado, G., y Hodge, B. (2017) Metodologías semióticas para análisis de la complejidad, Australia, Ed. Institute for Culture and Society, Western Sydney University.

Escobar, A. (2016) Autonomía y diseño. La realización de lo comunal, Popayú, Colombia: Ed. Universidad de Cauca.

Flusser, V. (2002) Filosofía del diseño, España: Ed. Sintesis.

Freire, P. (2011) La importancia de leer y el proceso de liberación, México: Ed. Siglo XXI.

Freire, P. (2015) Pedagogías de los sueños posibles. Por qué docentes y alumnos necesitan reinventarse en cada momento de la historia. México: Ed. Siglo XXI.

García, R. (2000) El conocimiento en construcción. De las formulaciones de Jean Piaget a la teoría de sistemas complejos, Barcelona, España: Ed. Gedisa.

Han, B., (2018) Hiperculturalidad, España: Ed. Herder.

Latour, B. (2008) Reensamblar lo social. Una introducción a la teoría del actor-red, Buenos Aires, Argentina: Ed. Manantial.

Lotman, I. (1996) La semiosfera I. Semiótica de la cultura y del texto, Valencia, España: Ed. Frónesis Cátedra, Universidad de Valencia.

Luhmann, N. (2007) Introducción a la Teoría de Sistemas, México: Ed. Universidad Iberoamericana.

Lutnæs, E. (2019) Enmarcando el concepto Alfabetización en diseño para un público en general, en Perspectivas de investigación en la era de las Transformaciones, Conferencia de la Academia para la Gestión de la Innovación en el Diseño, Londres, Reino Unido. DOI:10.33114/admin.2019.01.224

Margolin, V. (2005) Las políticas de lo artificial. Ensayos y estudios sobre diseño, México, Ed. Designio.

Meillassoux, Q. (2015) Después de la finitud. Ensayo sobre la necesidad de la contingencia. Buenos Aires, Argentina: Ed. Caja Negra.

Morin, E., (1998) Introducción al pensamiento complejo, España: Ed. Gedisa.

Noë, A. (2010) Fuera de la cabeza, Por qué no somos el cerebro. Y otras lecciones de la biología de la consciencia, Barcelona, Ed: Kairós.

Norman, D. (2005) El diseño emocional, por qué nos gustan (o no) los objetos cotidianos, España: Ed. Paidós.

Simon, H. (1996) The sciences of the artificial [Las ciencias de lo artificial]. USA: Mit. Press, Cambridge, MA.

Souza de Freitas, A. (2015) Prefacio. Pedagogía de los sueños posibles: El arte de volver posible lo imposible en Pedagogías de los sueños posibles. Por qué docentes y alumnos necesitan reinventarse en cada momento de la historia. México: Ed. Siglo XXI.

Vargas, R. y Briede, J. (2020) Alfabetización de Diseño, o el Diseño de Individuos de Postindustria, en RChD: creación y pensamiento, 5(8). 63-76. DOI: 10.5354/0719837X.2020.57652.

Vygotsky, L. (2015) Pensamiento y lenguaje, México: Ed. Boocket.

Wittgenstein, L. (2008) Investigaciones filosóficas, Barcelona, España: Ed. Crítica.

Wong, W. (1991) Fundamentos del diseño Bi- y Tri-dimensional, Barcelona, España: Ed. Gustavo Gili. 


\begin{abstract}
This text seeks to contribute to the reflection on the interaction given between people througt the designed objects. Underlying this relationship is the mediation contribution that the design has, namely, dialectial. In this sense, reflecting on this implication that design has, is crucial and more so in today's time. In the same way, it will be essential to analyze the influence and intervention that desing has in all areas of life, influencing the experience, the experience and the varoius ways of life of people, promoting, conditioning and determining ways of life and the ways in which they relate to the environment, so it is important that project disciplines be understood as a conditioning form of human and social behavior. In this context, the existence of a "design literacy" (Lutnæs, 2019) is crucial as an underlying dialogue process, it is a social continuum. Thinking of design as a discipline aimed at training, informing, promoting, articulating, regulating, reordering, re-signifying or reinterpreting decisively contribuyes to the social action involved, providing value and power to the interaction between people.

In order for this dialogical process to take place, it is important to keep in mind the context in which these interactions occur, for this, to think of a "semiosphere (Lotman, 1996) of the interactions, it's an opportunity to analyze the various resulting dialogues between people and what is designed, thus producing a doublé "analogical hermeneutical" process (Beuchot, 2016), the first one given when the designer performs all the actions required and involved to arrive at a design response, the second moment occurs once the design is designed, it's immersed within the social context, generating an interaction with people.
\end{abstract}

Keywords: Project discipline -Literacy - Interaction - Dialogic process - Interpretation

Resumo: Este texto busca contribuir para la reflexão sobre a interação que se dá entre as pessoas por meio dos objetos projtados. Subjacente a esta relação está o contributo da mediação que o design tem, nomeadamente, a dialéctica. Nesse sentido, refletir sobre essa implicação que o design ten, é crucial e ainda mais nos días de hoje. Da mesma forma, será imprescindível analizar a influencia e intervenção que o design tem em todas as áreas da vida, influenciando a vivência, a vivência e os varios modos de vida das pessoas, promovendo, condicionando e determinando modos e modos de vida nos quais se relacionam com o meio ambiente, por isso é importante que as disciplinas de projeto sejam entendidas como uma forma condicionante do comportamento humano e social. Neste contexto, a existencia de uma "literacia design" (Lutnæs 2019) é crucial como um proceso de diálogo subjacente, é un continuum social. Pensar o design como uma disciplina que visa formar, informar, promover, articular, regular, reordenar, ressignificar ou reinterpretar contibui de forma decisiva para a ação social envolvida, valorizando e potenciando a interação entre as pessoas.

Para que este processo dialógico aconteça, é importante ter em mente o contexto em que esas interações acontecem, para isso, pensar em uma "semiesfera" (Lotman, 1996) das interações, é uma oportunidade de analizar os diversos diálogos resultantes entre as pessoas e o que é projtado, produzindo assim um duplo proceso "hermenêutico analógico" 
(Beuchot, 2016), o primeiro dos quais ocorre quando o designer executa todas as ações necessárias e implícitas para chegar a uma resposta do design, o O segundo momento ocurre uma vez que o design é desenhado, ele está imerso no contexto social, gerando uma interação com as pessoas.

Palavras chave: Disciplina de projrcto - Alfabetização - Interação - Processo dialógico - Interpretação

[Las traducciones de los abstracts fueron supervisadas por el autor de cada artículo] 